\title{
Coherent Synchrotron Radiation in Laboratory Accelerators and the Double-Spectral Feature in Solar Flares
}

\author{
Wellington Cruz ${ }^{1}$, Sérgio Szpigel ${ }^{1}$, Pierre Kaufmann ${ }^{1}$, \\ Jean-Pierre Raulin ${ }^{1}$ and Michael Klopf ${ }^{2}$ \\ ${ }^{1}$ CRAAM, Mackenzie University, 01302-907, São Paulo, Brazil \\ ${ }^{2}$ Institute of Radiation Physics, Helmholtz-Zentrum, Dresden, Germany
}

\begin{abstract}
Recent observations of solar flares at high-frequencies have provided evidence of a new spectral component with fluxes increasing with frequency in the sub-THz to THz range. This new component occurs simultaneously but is separated from the well-known microwave spectral component that maximizes at frequencies of a few to tens of $G H z$. The aim of this work is to study in detail a mechanism recently suggested to describe the double-spectrum feature observed in solar flares based on the physical process known as microbunching instability, which occurs with high-energy electron beams in laboratory accelerators.
\end{abstract}

Keywords. Solar flares, Coherent synchrotron radiation, Microbunching instability

\section{Introduction}

Several events observed at high-frequencies ( 0.2 and $0.4 \mathrm{THz})$ using the Solar Submillimeter Telescope (SST) at the El Leoncito Observatory in the Argentinean Andes have shown clear evidence of a new spectral burst component with fluxes increasing with frequency in the sub-THz range (Kaufmann et al. 2002; Kaufmann et al. 2004; Silva et al. 2007; Fernandes et al. 2016). This new component occurs simultaneously but is distinct from the well-known microwave spectral component that maximizes at frequencies of a few to tens of $\mathrm{GHz}$. More recently, impulsive bursts have been observed in the mid-infrared at $30 \mathrm{THz}$ using small telescopes with a relatively simple optical setup at the El Leoncito Observatory and at Mackenzie Presbyterian University in São Paulo, exhibiting fluxes considerably larger than those measured for the concurrent microwave and sub-THz frequencies (Kaufmann et al. 2013; Kaufmann et al. 2015; Miteva et al. 2016).

A number of interpretations based on different emission mechanisms have been suggested to explain the THz spectral component (see Krucker et al. (2013) for a detailed review), but none of them account for the microwave spectral component which is simultaneously observed. An alternative possibility which has been recently investigated (Kaufmann \& Raulin 2006; Klopf et al. 2010; Klopf et al. 2014) is that both spectral components can be produced by a single beam of accelerated electrons undergoing the process known as microbunching instability, which occurs in laboratory accelerators (Williams 2002). Such a process is responsible for the production of synchrotron radiation with a double-spectrum structure similar to that observed in several solar flares, showing a broadband coherent synchrotron radiation (CSR) component and a distinct incoherent synchrotron radiation (ISR) component with maximum at higher frequencies. 


\section{Microbunching Instability and the ISR/CSR Mechanism}

Extremely bright photon beams in the form of ISR are produced in laboratory accelerators by highly relativistic electrons moving through a dipole magnet. Several techniques have been developed to further enhance the brightness of the ISR photon beam (Friedman \& Herndon 1973), such as the use of periodic magnetic structures known as insertion devices (wigglers or undulators).

Under certain conditions, instabilities due to inhomogeneities of the magnetic field or wave-particle interactions, which arise from the feedback between the insertion device, the radiation field and the accelerated electron beam, can produce modulations of the density of electrons, generating spatial structures called microbunches (Williams 2002). At wavelengths comparable to or longer than the size of the microbunch, the near field of the radiation from each electron overlaps the entire microbunch structure, resulting in a multiparticle coherent interaction which produces the emission of broadband CSR.

The spectrum of synchrotron radiation emitted by a microbunch of highly relativistic electrons accelerated in a dipole magnetic field is derived by generalizing the results obtained from the classical theory of electrodynamics for a single radiating electron to a system with multiple electrons. For a monoenergetic electron beam with discrete microbunches, the spectral intensity (energy radiated per unit of solid angle per unit of frequency) emitted by a microbunch containing $N_{e}$ electrons is given by (Williams 2002):

$$
\frac{d^{2} W}{d \nu d \Omega}=\left\{N_{e}[1-f(\nu)]+N_{e}^{2} f(\nu)\right\} I_{e}(\nu)
$$

where $I_{e}(\nu)$ is the single-electron spectral intensity for synchrotron emission at frequency $\nu$ and $f(\nu)$ is a form factor defined from the size and the shape of the microbunch structure, which is given by the square of the Fourier transform of the normalized longitudinal spatial charge distribution $S(z)$ within the microbunch structure,

$$
f(\nu)=\left|\int_{-\infty}^{\infty} \exp [i 2 \pi \nu(\hat{n} \cdot z) / c] S(z) d z\right|^{2} .
$$

For a gaussian-shape microbunch structure with a characteristic length scale $\sigma_{b}$, as it is often assumed in a laboratory accelerator scenario, the form factor is given by $f(\nu)=\exp \left[-4 \pi^{2} \tau_{b}^{2} \nu^{2}\right]$, where $\tau_{b}=\sigma_{b} / c$ is a characteristic time-width scale. One should note that in a solar flare accelerator scenario the longitudinal spatial charge distribution $S(z)$ may be far more complex than the gaussian function usually assumed in a laboratory accelerator. Simulations considering different analytical solutions for $S(z)$ were tested (Klopf et al. 2010; Klopf et al. 2014), showing that particularly good fits can be obtained using hyperbolic secant-shape microbunch structures, for which the form factor is given by $f(\nu)=\operatorname{sech}\left[\pi \tau_{b} \nu / 2\right]$.

\section{Simulations of the ISR/CSR mechanism for THz burst events}

In the simulations of the ISR/CSR mechanism presented here, we have considered a beam of accelerated electrons with kinetic energies in the range from $E_{\min }$ to $E_{\max }$ following a power-law distribution $n(E)=A E^{-\delta}$, where $\delta$ is the spectral index and $A$ is a normalization constant such that $\int n(e) d E=1$. We have also considered that only electrons with kinetic energies above a certain threshold $E_{t h}$ (set at a few $M e V$ ) can form microbunch structures because of Coulomb repulsion (Ingelman \& Siegbahn 1998). In this way, we assume that the number of electrons participating in the CSR process is just a fraction $x$ of the number of high-energy electrons, i.e. $N_{C S R}=x N_{h i g h}$. 

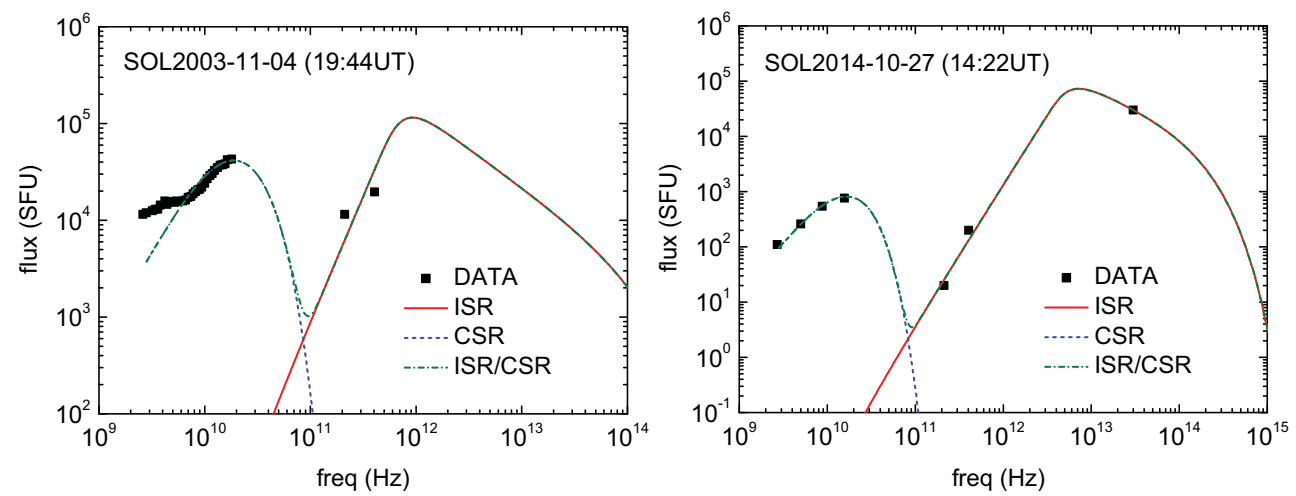

Figure 1. Fits to the spectra of the solar flares SOL2003-11-04 $\left(N_{e}=1.0 \times 10^{35} ; E_{\text {min }}=50 \mathrm{keV}\right.$; $E_{\text {max }}=100 \mathrm{MeV} ; \delta=2.5 ; \phi_{s}=0.52^{\prime \prime} ; n_{p}=10^{9} \mathrm{~cm}^{-3} ; B=1000 \mathrm{G} ; \theta=60^{\circ}$; $\left.N_{C S R} / N_{h i g h}=5.2 \times 10^{-15} ; E_{t h}=5.0 \mathrm{MeV} ; \tau_{b}=67 \mathrm{ps}\right)$ and SOL2014-10-27 $\left(N_{e}=1.3 \times 10^{35} ;\right.$ $E_{\text {min }}=100 \mathrm{keV} ; E_{\max }=100 \mathrm{MeV} ; \delta=2.3 ; \phi_{s}=0.031^{\prime \prime} ; n_{p}=10^{9} \mathrm{~cm}^{-3} ; B=1000 \mathrm{G}$; $\left.\theta=60^{\circ} ; N_{C S R} / N_{h i g h}=1.2 \times 10^{-17} ; E_{t h}=5.0 \mathrm{MeV} ; \tau_{b}=80 \mathrm{ps}\right)$. The individual contributions from the ISR and the CSR components are also shown.

In Figure 1 we show the fits to the spectra of the solar flares SOL2003-11-04 (19:44UT) and SOL2014-10-27 (14:22UT), from microwave to $T H z$ frequencies, obtained through simulations of the ISR/CSR mechanism considering a hyperbolic secant-shape microbunch structure. The model parameters adjusted to fit the spectra are: the number of accelerated electrons $N_{e}$, the spectral index $\delta$, the minimum electron kinetic energy $E_{m i n}$, the maximum electron kinetic energy $E_{\max }$, the source angular size $\phi_{s}$, the viewing angle $\theta$, the magnetic field strength $B$, the plasma density $n_{p}$, the fraction of high-energy electrons participating in the CSR process $N_{C S R} / N_{h i g h}$, the energy threshold for microbunch formation $E_{t h}$ and the characteristic time-width scale of the microbunch structure $\tau_{b}$.

\section{Conclusion}

We have shown through numerical simulations that the ISR/CSR mechanism can provide a plausible explanation to the double-spectrum observed for several solar flares. Using typical flaring parameters and power-law energy distributions, we have obtained remarkable good fits to the spectra of the solar flares SOL2003-11-04 and SOL2014-10-27.

\section{References}

Kaufmann, P. et al. 2002, Astrophys. J., v. 574, p. 1059-1065

Kaufmann, P. et al. 2004, Astrophys. J. Lett., v. 603, n. 2, p. L121

Silva, A. V. R. et al. 2007, Sol. Phys., v. 245, p. 311-326

Fernandes, L. O. T. et al. 2016, ASP Conf. Series, v. 504, p. 87

Kaufmann, P. et al. 2013, Astrophys. J., v. 768, p. 134

Kaufmann, P. et al. 2015, J. Geophys. Res.: Space Phys., v. 120, n. 6, p. 4155-4163

Miteva, R. et al. 2016, Astron. Astrophys., v. 586, p. A91

Krucker, S. et al. 2016, Astron. Astrophys., v. 21:58

Kaufmann, P. \& Raulin, J.-P. 2006, Phys. Plasmas, v. 13, 070701

Klopf, J. M., Kaufmann, P., \& Raulin, J.-P. 2010, Bul. Am. Astron. Soc., v. 42, p. 905

Klopf, J. M., Kaufmann, P., Raulin, J.-P., \& Szpigel, S. 2014, Astrophy. J., v. 791, n. 1, p. 31

Friedman, M. \& Herndon, M. 1973, Phys. of Fluids, v. 16, p. 1982-1995

Williams, G. P. 2002, Rev. Sci. Instr., v. 73, p. 1461-1463

Ingelman, G. \& Siegbahn, K. 1998, Fysik-Aktuellt,v. 1, p. 3 